\title{
Long-tailed distributions and non-uniform expansion in the analysis of large-scale structure
}

\author{
L.S. Schulman ${ }^{\mathrm{a}, \mathrm{b}, *}$ \\ ${ }^{a}$ Physics Department, Clarkson University, Potsdam, NY 13699-5820, USA \\ ${ }^{\mathrm{b}}$ Chemistry Department, Columbia University, New York, NY 10027, USA
}

Received 15 September 1996

\begin{abstract}
This article presents two caveats for the analysis of large-scale structure. Within distances for which the fractal structure of the distribution of galaxies obtains, fluctuations may be on the scale of the measurements themselves, as is typical of fractals. Second, within this region it is doubtful that the asymptotic recession velocity has been reached. Given the evidence of fractal structure to large distances, these caveats have serious implications for measurements of the Hubble constant.
\end{abstract}

PACS: 98.65.Dx; 98.62.Py; 05.40.+j; 02.40.+m

Keywords: Large-scale structure; Hubble constant; Fractals

\section{Introduction}

This article is concerned with certain pitfalls of data analysis in the study of largescale structure, in particular, those related to the apparently fractal structure of a neighborhood of our galaxy [1,2]. Perhaps, the most important single quantity that one wishes to deduce is the Hubble "constant," $H_{0}$, and, as is well known, there are widely varying values that vie for acceptance. We argue that this is to be expected for fractal structures - in fact, data analysis for fractals is subject to a bias in which one may be tempted to discard highly deviant data, but should not. A second issue is the non-uniform expansion of the universe. This has been treated theoretically $[3,4]$, but does not lead to definite predictions because of uncertainty in the energy distribution. Our present considerations suggest that even at considerable distance recession velocities may be far from their asymptotic values. In this article we do not support any

* Corresponding author. E-mail: schulman@polaris.clarkson.edu. 
particular value of $H_{0}$; rather we discuss the implications of fractality on its measurement and perhaps soften the impact of the apparent contradictions. We explain the existence of discrepancies; we do not resolve them.

Similar concerns were expressed in Ref. [5]. Turner et al. considered the possibility that $H_{0}$ possess a wide spread in values (as measured from different galaxies) because of fluctuations in the distance/velocity distribution function. Support for this idea was found in the output of many-body simulations under various cosmological scenarios. Here we show the roots of these deviations to be general. So long as the mass/energy distribution is fractal, one expects large deviations and associated phenomena. In Ref. [6] a similar point is made in dealing with what may have appeared to be a contradiction between fractal structure at large scales and apparent homogeneity in some small samples. There they attribute the problem to small-scale effects, a characteristic property of fractals.

We also propose ways to deal with some problems. One is an extension of Ref. [5] in which simulations could suggest the form of distribution functions. Another is to use a statistic other than the average to estimate $H_{0}$. It is also possible that selection processes in the analysis of data are already effectively choosing another statistic, accounting for the small observational scatter that contradicts both our expectations and those of Ref. [5].

\section{Long-tailed distribution}

The evidence for fractal structure in a large swath of the observed universe is good, although whether and where large scale structure goes over to a homogeneous distribution is a more contentious issue. What I emphasize in this article is the associated implication that distribution functions will be long-tailed. This can allow a new form of bias to enter in the selection of data.

For example, consider the function $P(n ; V)$, the probability that a given volume, $V$, contains $n$ galaxies. For a homogeneous distribution, this is $P_{\text {Poisson }}(n ; V)=(\rho V)^{n}$ $\exp (-\rho V) / n$ !, with $\rho$ an average number-density. For large $\rho V$ this goes to a Gaussian centered on $\rho V$, with variance, $\sqrt{\rho V}$, that is much less than $\rho V$. On the other hand, the distribution function for fractals can be far from Poissonian. ${ }^{1}$ A numerical study of this phenomenon was undertaken in [8]. Although the function $P(0 ; V)$ is essential to the box-counting definition of fractals, $P(n ; V)$, for $n \neq 0$, is not determined by the fractal dimension alone. For example, consider the Cantor set with a lower cutoff, $\{\xi=$ $\left.\sum_{k=1}^{N} a_{k} / 3^{k} \mid a_{k}=0,2\right\}$. If the volumes are intervals of length $2^{-M}(M<N)$, and they coincide with breakup-thirds of the Cantor construction, then they will either contain $2^{N-M}$ points, or none at all. Randomizing the volumes or the Cantor construction will avoid this extreme. It is clear, however, that not only are the distributions arising

\footnotetext{
${ }^{1}$ Mathematically, to define $P(n ; V)$ on a fractal set there must be a lower size cutoff. For physical applications this arises naturally. Through its dropoff properties with $n$, the function $P(n ; V)$ may also provide a handle on lacunarity [7].
} 
from fractals long-tailed, but they can have most of the weight in the tail. (In Ref. [8] intermediate cases are considered.) It is also clear that these properties of $P(n ; V)$ are not peculiar to the Cantor set, but hold for any set with hierarchical structure. (Note, by the way, that even with Poisson statistics, there is still a likelihood that somewhere there is a large void. That is, by not selecting on a specific $V$, one will have a reasonable chance of finding large empty regions, but they will be far less common than they are for a fractal, see Ref. [9].)

For the purpose of making deductions about systems characterized by long-tailed distributions, an important caveat is that the distributions may be non-self-averaging or weakly self-averaging. For a random variable, $X$, one expects that averaging $N$ measurements will better estimate the true average than will a single measurement. But for long-tailed distributions this need not be the case! Let $A_{N} \equiv(1 / N) \sum_{k=1}^{N} X_{k}$, where $X_{k}$ are i.i.d. random variables with the distribution of $X$. For a finite-variance random variable, the variance of $A_{N}$ is $1 / N$ times the variance of $X$, so you improve your estimate with more measurements. For long-tailed distributions the variance does not exist, and for some of them the average does not exist either.

I now discuss the Lévy distributions, [10,11], which are the "Gaussians" of long-tailed distributions. They are characterized by having Fourier transform $\exp \left(-\lambda|k|^{\alpha}\right)$ with $0<\alpha<2$ and $\lambda$ a parameter, which yields probability densities $p(x) \sim 1 /|x|^{\alpha+1}$ for large $|x|$. Because moments may not exist, I measure the "self-averaging" property in terms of the spread in the distribution of $A_{N}$ about its central value, compared to the corresponding spread for $X$. Let the distribution function for $X$ be $p_{X}(x)$, which for simplicity is taken to be symmetric about zero. Define

$$
W(\mu) \equiv \int_{-\mu}^{\mu} p_{X}(x) d x
$$

which is the probability that a measurement of $X$ will yield a result within $\mu$ of zero. I compare this to

$$
W^{N}(\mu) \equiv \operatorname{Pr}\left(-\mu<\frac{1}{N} \sum_{k=1}^{N} X_{k}<\mu\right)=\int_{-\mu}^{\mu} p_{A_{N}}(x) d x .
$$

If $\phi_{X}$ is the characteristic function of $X$, it is immediate that $W^{N}(\mu)=\int_{-\mu N}^{\mu N} d x \int d k$ $\times \exp (-i k x) \phi_{X}^{N}(k) / 2 \pi$. For the Lévy distributions, this allows the $N$-dependence of $W^{N}$ to be derived explicitly:

$$
W^{N}(\mu)=\int_{-\mu N}^{\mu N} d x \frac{1}{2 \pi} \int d k e^{-i k x} e^{-N \lambda|k|^{\alpha}}=\int_{-\mu N^{1-1 / x}}^{\mu N^{1-1 / x}} d x p_{X}(x) .
$$

(The second equality uses the rescaling $|q|^{\alpha}=N|k|^{\alpha}$.) For the Cauchy distribution, $\alpha=$ 1 , there is no change. For $\alpha>1$, with enough measurements $W^{N}$ becomes narrower, but for small $\alpha-1$ one can expect large deviations. 


\subsection{Fluctuations}

Although variance is not available as a measure of fluctuations in long-tailed distributions, other criteria may be applied. For example, for a finite sample (as for galaxies), one can compare typical values and maximal values. As we have seen for the function $P(n ; V)$ for the truncated Cantor set, the fluctuations - differences between largest and smallest values - are, in fact, the typical measurements.

In the analysis of data for large scale structure an important function is $\operatorname{Pr}(v \mid r)$, the probability that a galaxy at a distance $r$ has velocity $v$. With the realization that this is likely to be long-tailed, several caveats arise:

(1) Since large fluctuations are typical of long-tailed distributions, one must be careful in editing deviant observations. In a sense, one may know too much about a given galaxy. One may understand the special circumstances giving rise to, say, an extremely high velocity, and eliminate this galaxy from the data set as being atypical. For long-tailed distributions, the atypical is the norm, and such editing is a form of bias.

(2) Similar considerations apply to the correction made with respect to the high velocities in the direction of mass concentrations such as the Great Attractor. One is presented with an extreme overdensity and corrects for infall in order to deduce the residual and "true" expansion velocity. (This is a major correction in Ref. [12].) In dealing with fractal structures large deviations at all scales are the norm. For example, if one is interested in density fluctuations on a critical percolation cluster, the larger the scale, the larger the fluctuation. Another way to say this is that the overall velocity of even a large supercluster may itself be significantly different from that of the background radiation frame.

\section{Non-uniform material expansion}

One would like to know when one has reached a distance scale where velocity and position have the proportionality characteristic of a uniformly expanding cosmology, and in this way deduce $H_{0}$. As discussed above, one cannot expect this to happen at distances where fractal behavior is manifested. In statistical mechanics, one is used to dealing with pseudo-fractality at one distance scale, and passing to homogeneity at a larger scale. (For example, a percolating cluster at $p$ just above $p_{c}$ will have correlation lengths $\xi \sim\left|p-p_{c}\right|^{-\nu}$. For samples of linear dimension $L>\xi$, at the largest scales there will be homogeneity, while below $\xi$ the system will look like a critical cluster. The transition from power law to exponential cumulants is via a two-point function of the form $\exp (-r / \xi) / r^{\text {[power] }}$, see Ref. [13].)

The non-uniform nature of material expansion has been appreciated in several contexts. In Ref. [14] the expansion of the universe is compared to that of a balloon with coins glued to its surface. Bound systems, like the solar system do not participate in the global expansion. The model in Ref. [14] was based on Ref. [3]. More recently, 
this question was taken up in Ref. [4]. From Ref. [3] it is clear that theory alone cannot determine the systematic growth of average expansion velocity with distance. Although the general relativistic calculation is more complicated than the corresponding Newtonian one, [3] finds that Newtonian intuition can provide a guide. The essential issue is whether the average density within a region is larger or smaller than the overall average density. Since we do not know where the mass is, we cannot predict one way or the other. On the basis of luminosity alone, one would again say that so long as fractal behavior is manifested one should not expect to have reached the asymptotic recession-velocity. (For a true fractal, a non-empty volume will always have an elevated local density, since the global density is zero.)

Given this inability to make firm theoretical predictions, I turn to observation; not to specific structure observations but to deductions about expansion based on those observations. Specifically, I take up predictions about the systematic dependence of expansion on distance that are implicit in the arguments of Ref. [15].

In Ref. [15] a mechanism was proposed for the formation of a non-uniform galaxy distribution, replete with voids, clusters and hierarchical structure. That mechanism yielded a two-point correlation function with a $1 / r$ dropoff (to be contrasted to the observed $1 / r^{\gamma}$ with $\gamma \approx 1.8$ ). Since the formation process is expected to have occurred at an early epoch the issue of non-uniform expansion needed to be confronted. For the distribution of galaxies there seems to be no distance scale between the smallest (the size of a galaxy) and the largest (the transition distance to homogeneity, or if that does not exist, the scale of the universe). ${ }^{2}$ Therefore, since gravity has no distance scale a power law at one epoch should become a power law at another. This argument was consistent with experience at that time with $N$-body simulations (and still is). These suggested that under the influence of gravity there was an increase as a function of time in the exponent in the two-point correlation function. Call the two-point function $g(r)$. In a plot of $\log g(r)$ versus $\log r$ the initial distribution is a straight line of slope -1 in the fractal region. (This goes over to slope- 0 in the homogeneous region.) In the present epoch that same plot gives a slope of $-\gamma$. If indeed scale invariance implies that a straight line goes over to a straight line, then conservation of matter (on the average) allows a prediction to be made about the time of galaxy formation (see Ref. [15]).

My goal here is not to consider the specific galaxy formation process, but to check the implications of the scale-independence arguments for the average expansion as a function of distance. Suppose that on the average (which we here assume exists.) the material at a distance $r$ goes to another distance, $f(r)$. Conservation of the material in

\footnotetext{
${ }^{2}$ Although individual galaxies are also dominated by gravity, hierarchical structure and scale invariance need not persist internally. One can encounter new distance scales constructed from characteristic angular momenta.

${ }^{3}$ A power law dropoff necessarily includes a quantity with dimensions of distance, e.g., $g(r) \sim r_{0} / r$. Our argument about scale invariance relates not to $r_{0}$, but to the lack of a break in the $\operatorname{linear} \log g(r)$ vs. $\log r$ plot.
} 
the shell $r$ to $r+d r$ implies that $g_{\text {old }}(r) r^{2} d r=g_{\text {new }}(f) f^{2} d f$. If the new and old $g$ s have exponents 1 and $\gamma$, respectively and if $f(0)=0$, then it is easy to see that

$$
f(r)=\text { const } r^{2 /(3-\gamma)} \approx \text { const } r^{5 / 3} \quad \text { with } r<\text { scale of homogeneity. }
$$

This implies a considerable degree of non-uniformity, both in position and velocity. Incidentally, without the mechanism proposed in Ref. [15] for an initial $1 / r$ correlation function, if one were to start from a homogeneous distribution, the power of $r$ in the above equation would not be $5 / 3$ but $5 / 2$.

There are ways in which the foregoing argument can break down. For one thing, there could be large distance scales, although the validity of the power law dropoff - with the same value of $\gamma$ - for both galaxies and for clusters of galaxies suggests otherwise, up to the distances to which this relation holds. Furthermore, at some stage general relativistic effects must enter, affecting both the description of fractals and the definition of "distance", $r$ (see Ref. [16] for related issues).

My point though is not that the argument holds at all distance scales, only that it suggests that the distance scale for "full participation in the expansion of the universe" may be large. Moreover, to the extent that within this scale there is evidence of fractal structure (presumably much of it gravitationally induced) it is not possible to reliably separate peculiar and general recession velocities.

\section{Suggestions for data analysis}

Although the main message of this article is caution, we will make suggestions for a more rigorous data analysis. There is now a considerable body of simulations of galactic dynamics [17]. Each such simulation can provide a conditioned distribution function for velocity, $\operatorname{Pr}(v \mid r)$. As mentioned above, Ref. [5] used this approach to estimate expected scatter in measurements of $H_{0}$. The problem of missing mass casts an overall uncertainty on the results, but this is a problem already addressed [17]. For example, in Ref. [5] a variety of cosmological situations are considered. One could also look at the observed distribution functions for the galaxies, although one would need to be confident in both distance and velocity values and be confident as well that no data are missing from the tail of the distribution. Thus, the usual issues of bias and selection arise all the more seriously because of the desire to include tail events.

A study of $\operatorname{Pr}(v \mid r)$ could have several possible outcomes. Perhaps it will turn out that despite the fact that the simulation recovers fractal structure the function does not have a long tail. Alternatively, finding a long tail of a particular sort would indicate the size and distance scale needed for a reliable statistical analysis.

A further technique (especially useful if the methods above give an a priori idea of what distribution to expect) is to use a statistic other than the average. When one makes a least squares fit to a straight line, the slope is a linear function of the random "dependent" variable. Thus if this dependent variable is a set of measurements from a long-tailed distribution it will have large fluctuations. However, consider for example, 
the middle value of three measurements. It is easy to see ${ }^{4}$ that if one measurement would fall like $1 / x^{1+\alpha}$, the middle of three such would have a tail going like $1 / x^{1+2 \alpha}$. If this is still too long, use the middle of five measurements, etc. Now it is likely that the informal "editing" of data that I discussed earlier will have discarded some of the tail and made the actual data analysis closer to what I now suggest.

\section{Conclusions and discussion}

Our message is summarized by two caveats. Within distances for which the fractal structure of the distribution of galaxies obtains, fluctuations of density and other quantities may be expected to be on the scale of the measurements themselves, as is typical of fractals. As such, "corrections" for systematic motion, such as "infall velocity" with respect to local large aggregates, do not provide reliable indications of global quantities. In fact, related selection of data can represent a significant bias not previously recognized. Second, the asymptotic recession velocity for the expansion of the universe, cannot be deduced so long as one does not know when asymptopia sets in. We further suggest that such asymptotic behavior may not obtain within locally fractal regions.

In Ref. [18] Peebles comments that the "Hubble law" was originally based on a nearby sample (redshift velocity $\leqslant 1000 \mathrm{~km} \mathrm{~s}^{-1}$ ), where one now recognizes there to be considerable inhomogeneity, hence no a priori expectation of a linear distance-velocity relation. The present article emphasizes the role of inhomogeneity out to greater distances, from both the statistical and dynamical points of view. It is thus remarkable that observational support for a linear relation continues to be good (notwithstanding puzzles connected to collective velocity corrections, cf. pp. 85-88, in Ref. [18]). For example, in Ref. [19] attempts were made to find variation in $H_{0}$ along the lines suggested in Ref. [5]. The authors concluded "that $H_{0}$ measured globally is constant to $\pm 7 \%$ from $3000 \mathrm{~km} \mathrm{~s}^{-1}$ to $15000 \mathrm{~km} \mathrm{~s}^{-1}$." One possibility is that the overall energy density is more homogeneous than the luminosity distribution. This would not however explain the smallness of the deviations from the straight line exhibited by some data sets.

\section{Acknowledgements}

I wish to thank J.L. Anderson, R. Bidaux, B.B. Mandelbrot and L.J. Schulman for helpful discussions. This work was supported in part by the United States National Science Foundation grant PHY 9316681.

\footnotetext{
${ }^{4}$ The distribution of the middle of three i.i.d. random variables is $6 \operatorname{Pr}\left(X_{2}=u \mid X_{1}<X_{2}<X_{3}\right)=$ $6 p(u) \int_{-\infty}^{u} p\left(u^{\prime}\right) \int_{u}^{\infty} p\left(u^{\prime \prime}\right)$. For large positive $u$ one of these integrals gives 1 and the other $1 / u^{\alpha}$.
} 


\section{References}

[1] L. Pietronero, Physica A 144 (1987) 257.

[2] P.H. Coleman, L. Pietronero, Phys. Rep. 213 (1992) 311.

[3] P.D. Noerdlinger, V. Petrosian, Astrophys. J. 168 (1971) 1.

[4] J.L. Anderson, Phys. Rev. Lett. 75 (1995) 3602.

[5] E.L. Turner, R. Cen, J.P. Ostriker, Astron. J. 103 (1992) 1427.

[6] F. Sylos Labini, A. Gabrielli, M. Montuori, L. Pietronero, Physica A 226 (1996) 195.

[7] B.B. Mandelbrot, A. Vespignani, H. Kaufman, Europhys. Lett. 32 (1995) 199.

[8] H.K. Harding Jr., D. Lange, L.S. Schulman, Counting-Fluctuations for Fractal Sets, CISP Report, 1995, unpublished.

[9] H.D. Politzer, J.P. Preskill, Phys. Rev. Lett. 56 (1986) 99.

[10] E.W. Montroll, B.J. West, On an enriched collection of stochastic processes, in: E.W. Montroll, J.L. Lebowitz, (Eds.), Fluctuation Phenomena, North-Holland, Amsterdam, 1987.

[11] J. Klafter, M.F. Shlesinger, G. Zumofen, Phys. Today 49 (2) (1996) 33.

[12] W. Freedman, B. Madore, J. Mould, L. Ferrarese, R. Hill, R. Kennicutt, A. Saha, P. Stetson, J. Graham, H. Ford, J. Hoessel, J. Huchra, S. Hughes, G. Illingworth, Nature 371 (1994) 757.

[13] M.E. Fisher, J. Math. Phys. 5 (1964) 944.

[14] C.W. Misner, K.S. Thorne, J.A. Wheeler, Gravitation, Freeman, San Francisco, 1973.

[15] L.S. Schulman, P.E. Seiden, Astrophys. J. 311 (1986) 1.

[16] M.B. Ribeiro, Astrophys. J. 388 (1992) 1.

[17] See, for example, D.W. Latham, L.A. Nicolaci da Costa, Large-Scale Structures and Peculiar Motions in the Universe, Astro. Soc. Pacific, Conf. Ser., vol. 15, Astronomical Society of the Pacific, 1991.

[18] P.J.E. Peebles, Principles of Physical Cosmology, Princeton Univ. Press, Princeton, 1993.

[19] T.R. Lauer, M. Postman, Astrophys. J. 400 (1992) L47. 